IFUM-FT-671

\title{
Noncommutative $\mathcal{N}=1,2$ super $U(N)$ Yang-Mills: $\mathrm{UV} / \mathrm{IR}$ mixing and effective action results at one loop
}

\author{
Daniela Zanon \\ Dipartimento di Fisica dell'Università di Milano and \\ INFN, Sezione di Milano, Via Celoria 16, 20133 Milano, Italy
}

\begin{abstract}
Noncommutative $\mathcal{N}=1$ and $\mathcal{N}=2$ supersymmetric Yang-Mills theories with gauge group $U(N)$ are studied here using the background field method and superspace background covariant $D$-algebra in perturbation theory. At one loop divergences arise only in the two-point functions. They are logarithmic UV/IR divergences in the planar/nonplanar sectors that play a role dual to each other.

We consider contributions to the effective action with vector external lines. We find that the three-point function vanishes, while the four-point function receives contributions both from vector and from chiral matter loops.
\end{abstract}

e-mail: daniela.zanon@mi.infn.it 
Recently noncommutative geometry received much attention both in string theory and in gauge field theory [1, 2, 3, 4, 5], and various aspects of the intimate connection between the two approaches have been enlightened.

In this paper we restrict our attention to noncommutative field theories and continue the study of the effective action for supersymmetric Yang-Mills theories in superspace perturbation theory. In [6, 7] the theory under consideration has been $\mathcal{N}=4$ supersymmetric Yang-Mills with gauge group $U(N)$. It is the 4-dimensional gauge field theory with the largest number of symmetries and as such the simplest to be studied even in its noncommutative version. The analysis is simplified if one uses the superfield formulation 8] combined with the background field method [6]. With these techniques perturbative calculations become manageable and gauge invariance is kept under control. In [6, [] contributions to the one-loop effective action have been considered: the two- and three-point functions with external background vector fields are zero as it happens in the commutative case [9, 10, 11]. In addition the four-point function has been computed and shown to be in agreement with the field theory limit of corresponding string amplitudes [12]. The noncommutative theory is completely free of ultraviolet and infrared divergences, as expected since the corresponding commutative theory is conformally invariant. In the background field method approach used in [6, 7] the perturbative calculations of background vector $n$-point functions are particularly simple because loops with quantum chiral superfields need not be evaluated. It is a trivial matter to show that the three ghost fields completely cancel corresponding contributions from the three chiral matter superfields. After all $\mathcal{N}=4$ super Yang-Mills has to be simple.

As soon as we relax the symmetry and look at the corresponding theories with $\mathcal{N}=1$ and $\mathcal{N}=2$ supersymmetries the matter-ghost cancellation no longer occurs and more ingenuity is required in order to proceed and compute the one-loop higher-order functions. At the level of the two-point function one finds UV and IR divergences in the planar and in the non planar sectors respectively. This is the UV/IR mixing that signals a $\beta \neq 0$ in the corresponding commutative theory.

Here we apply the same techniques introduced in [6, 7] to the noncommutative $\mathcal{N}=1$ and $\mathcal{N}=2$ supersymmetric Yang-Mills theories and consider corrections to the one-loop effective action up to the four-point function.

The noncommutative $\mathcal{N}=1$ supersymmetric Yang-Mills theory is described in $\mathcal{N}=1$ superspace by the following classical action (with notations and conventions as in [1])

$$
S=\left.\frac{1}{4 g^{2}} \operatorname{Tr}\left(\int d^{4} x d^{2} \theta W^{\alpha} W_{\alpha}+\int d^{4} x d^{2} \bar{\theta} \bar{W}^{\dot{\alpha}} \bar{W}_{\dot{\alpha}}\right)\right|_{*}
$$

where $W^{\alpha}=i \bar{D}^{2}\left(e_{*}^{-V} * D^{\alpha} e_{*}^{V}\right)$ is the gauge superfield strength. The fields are Lie-algebra valued, e.g. $V=V_{a} T^{a}$, in the adjoint representation of $U(N)$. In (1) the symbol $\left.\right|_{*}$ indicates that the superfields are multiplied using the $*$-product defined as

$$
\left.\left(\phi_{1} * \phi_{2}\right)(x, \theta, \bar{\theta}) \equiv e^{\frac{i}{2} \Theta^{\mu \nu} \frac{\partial}{\partial x^{\mu}} \frac{\partial}{\partial y^{\nu}}} \phi_{1}(x, \theta, \bar{\theta}) \phi_{2}(y, \theta, \bar{\theta})\right|_{y=x}
$$


If, in addition to the vector real superfield $V$ we introduce a chiral matter superfield $\Phi$, we can construct a theory in which $\mathcal{N}=2$ supersymmetry is realized. The corresponding classical action describes $\mathcal{N}=2$ noncommutative Yang-Mills written in terms of $\mathcal{N}=1$ superfields

$$
S=\left.\frac{1}{g^{2}} \operatorname{Tr}\left(\int d^{4} x d^{4} \theta e^{-V} \bar{\Phi} e^{V} \Phi+\frac{1}{4} \int d^{4} x d^{2} \theta W^{\alpha} W_{\alpha}+\frac{1}{4} \int d^{4} x d^{2} \bar{\theta} \bar{W}^{\dot{\alpha}} \bar{W}_{\dot{\alpha}}\right)\right|_{*}
$$

The noncommutative theories in (11) and (3) can be quantized using the background field method [6], essentially adopting the same procedure as in the commutative case [9, [3].

For the gauge multiplet one performs a non linear splitting between the quantum prepotential $V$ and the background superfield, via covariant derivatives (in quantum chiral representation [1])

$$
\begin{aligned}
& \nabla_{\alpha}=e_{*}^{-V} * D_{\alpha} e_{*}^{V} \rightarrow e_{*}^{-V} * \nabla_{\alpha}^{B} e_{*}^{V} \\
& \bar{\nabla}_{\dot{\alpha}}=\bar{D}_{\dot{\alpha}} \rightarrow \bar{\nabla}_{\dot{\alpha}}^{B}
\end{aligned}
$$

In this way the external background enters in the quantum action implicitly in the background covariant derivatives through the connections

$$
\nabla_{\alpha}^{B}=D_{\alpha}-i \boldsymbol{\Gamma}_{\alpha} \quad \bar{\nabla}_{\dot{\alpha}}^{B}=\bar{D}_{\dot{\alpha}}-i \overline{\boldsymbol{\Gamma}}_{\dot{\alpha}} \quad \nabla_{a}^{B}=\partial_{a}-i \boldsymbol{\Gamma}_{a}
$$

and explicitly in the background field strength $\mathbf{W}_{\alpha}=\frac{i}{2}\left[\bar{\nabla}^{B \dot{\alpha}},\left\{\bar{\nabla}_{\dot{\alpha}}^{B}, \nabla_{\alpha}^{B}\right\}\right]$. Background covariant gauge-fixing introduces additional terms

$$
-\frac{1}{4 g^{2}} \operatorname{Tr} \int d^{4} x d^{4} \theta V *\left(\nabla_{B}^{2} \bar{\nabla}_{B}^{2}+\bar{\nabla}_{B}^{2} \nabla_{B}^{2}\right) * V+S_{F P}+S_{N K}
$$

with Faddeev-Popov action

$$
S_{F P}=\operatorname{Tr} \int d^{4} x d^{4} \theta\left[\bar{c}^{\prime} * c-c^{\prime} * \bar{c}+\frac{1}{2}\left(c^{\prime}+\bar{c}^{\prime}\right) *[V, c+\bar{c}]_{*}+\ldots\right]
$$

and Nielsen-Kallosh ghost action

$$
S_{N K}=\operatorname{Tr} \int d^{4} x d^{4} \theta \bar{b} * b
$$

In (7) we have used the notation $[A, B]_{*}=A * B-B * A$. The three ghosts $c, c^{\prime}$ and $b$ are background covariantly chiral superfields, i.e. $\bar{\nabla}_{B}^{\dot{\alpha}} c=\bar{\nabla}_{B}^{\dot{\alpha}} c^{\prime}=\bar{\nabla}_{B}^{\dot{\alpha}} b=0$. While the Faddeev-Popov ghosts do have interactions with the quantum $V$ fields, the NielsenKallosh ghost interacts only with the background. Therefore it contributes only at one loop. In the following we drop the suffix $B$ from the covariant derivatives since covariance is everywhere with respect to the background and no ambiguity can arise. 
After gauge-fixing the quadratic quantum $V$-action becomes

$$
S \rightarrow-\frac{1}{4 g^{2}} \operatorname{Tr} \int d^{4} x d^{4} \theta V *\left(\square-i \mathbf{W}^{\alpha} * \nabla_{\alpha}-i \overline{\mathbf{W}}^{\dot{\alpha}} * \bar{\nabla}_{\dot{\alpha}}\right) * V
$$

where $\square \equiv 1 / 2 \nabla^{a} \nabla_{a}$ is the background covariant Laplacian.

For the matter superfield $\Phi$ which appear in (3) the quantum-background splitting is a standard linear one

$$
\Phi \rightarrow \Phi+\Phi_{B}
$$

where $\Phi_{B}$ is the background external field. The superfields $\Phi$ and $\Phi_{B}$ are background covariantly chiral $\bar{\nabla}_{\dot{\alpha}} \Phi=0$.

Our final goal is to compute one-loop contributions to the effective actions of $\mathcal{N}=1$ and $\mathcal{N}=2$ Yang-Mills with external vector background fields. Therefore the corresponding actions quadratic in the quantum fields are all we need for these one-loop calculations. From (9) we see that the interactions with the background are at most linear in the $D$ spinor derivatives. Since at least two $D$ 's and two $\bar{D}$ 's are needed in the loop in order to complete the $D$-algebra, from (9) one starts obtaining a nonvanishing result at the level of the four-point function. We conclude that for the $\mathcal{N}=1$ theory the contributions to the vector two- and three-point functions can be generated only by ghosts circulating in the loop. For the $\mathcal{N}=2$ theory we have to take into account also the contributions from the chiral matter loop.

As mentioned above the three ghosts are background covariant chiral superfields with actions given in (7) and (8) respectively. Since we only compute up to one loop, in (17) we can drop terms that contain interactions with the quantum $V$-field. The same considerations apply also to the chiral superfield $\Phi$ in (河). In fact in our calculations we do not consider diagrams with external matter. Therefore in (3i) we keep only the quantum $\Phi$ 's and drop their interactions with the quantum $V$ 's that would never enter in a one-loop diagram.

For the $\mathcal{N}=1$ theory we end up with the sum of three identical ghost loops. Each ghost contributes to the one-loop effective action exactly as a standard background covariant chiral superfield would do, the only difference being an overall opposite sign because of the statistics. For the $\mathcal{N}=2$ theory we obtain contributions from covariantly chiral loops with a factor $(-3)$ from the ghosts and a factor $(+1)$ from the chiral matter. Thus we explain the general procedure for the computation of a chiral one-loop contribution. We use a formulation of the background covariant field method which is particularly simple and it is valid for real representations of the gauge group as it is the case of interest to us, i.e. the adjoint representation. We do not consider matter in complex representations which are in general affected by anomalies [14].

The rules of background covariant perturbation theory for commutative supersymmetric Yang-Mills are clearly explained in ref. [11] sect. 6.5. First we summarize here the part which is relevant for our specific calculation. Then we introduce the modifications needed in the noncommutative case. 
In order to obtain the Feynman rules for a general background covariantly chiral superfield $\Phi$ with action $S_{m}$ one starts from the generating functional

$$
Z(J, \bar{J})=\int \mathcal{D} \Phi \mathcal{D} \bar{\Phi} \exp \left[S_{m}+\left(\int d^{4} x d^{2} \theta J \Phi+\text { h.c. }\right)\right]
$$

where both $\Phi$ and $J$ are covariantly chiral $\bar{\nabla}^{\dot{\alpha}} \Phi=\bar{\nabla}^{\dot{\alpha}} J=0$. The one-loop contribution to the effective action from the $\Phi$ superfield is given by the Gaussian integral over the quantum matter fields

$$
\Delta=\int \mathcal{D} \Phi \mathcal{D} \bar{\Phi} \exp \left[\int d^{4} x d^{4} \theta \bar{\Phi} \Phi\right]
$$

The covariant generalization of the standard method leads to the introduction of chiral d'Alembertians $\square_{ \pm}$defined as

$$
\bar{\nabla}^{2} \nabla^{2} \Phi=\square_{+} \Phi \quad \nabla^{2} \bar{\nabla}^{2} \bar{\Phi}=\square_{-} \bar{\Phi}
$$

with

$$
\begin{aligned}
& \square_{+}=\square-\frac{i}{2}\left(\nabla^{\alpha} \mathbf{W}_{\alpha}\right)-i \mathbf{W}^{\alpha} \nabla_{\alpha} \\
& \square_{-}=\square-\frac{i}{2}\left(\bar{\nabla}^{\dot{\alpha}} \overline{\mathbf{W}}_{\dot{\alpha}}\right)-i \overline{\mathbf{W}}^{\dot{\alpha}} \bar{\nabla}_{\dot{\alpha}}
\end{aligned}
$$

In this way one finds [11] that

$$
\Delta=\exp \left[\int d^{4} x d^{2} \theta \frac{1}{2} \frac{\delta}{\delta J}\left[\bar{\nabla}^{2} \nabla^{2}-\bar{D}^{2} D^{2}\right] \frac{\delta}{\delta J}\right] \cdot \exp \left[-\int d^{4} x d^{2} \theta \frac{1}{2} J \square_{0}^{-1} J\right]
$$

where $\square_{0}=1 / 2 \partial^{a} \partial_{a}$ is the free d'Alembertian. A one-loop graph consists in a string of vertices and propagators, i.e. a vertex like $\left[\bar{\nabla}^{2} \nabla^{2}-\bar{D}^{2} D^{2}\right]$, a standard scalar propagator $\left(-\square_{0}^{-1}\right)$, a vertex again and so on. The calculation is more conveniently performed using the background chiral representation, $\bar{\nabla}_{\dot{\alpha}}=\bar{D}_{\dot{\alpha}}$. Indeed this allows to simplify all the vertices but one, making repeated use of the identity

$$
\left[\bar{\nabla}^{2} \nabla^{2}-\bar{D}^{2} D^{2}\right] \bar{D}^{2}=\left[\square_{+}-\square_{0}\right] \bar{D}^{2}
$$

The net result is that one has to compute one-loop diagrams constructed with the following propagators and vertices

$$
\begin{array}{lll}
\text { propagators : } & -\frac{1}{\square_{0}} & \\
\text { all vertices : } \quad \square_{+}-\square_{0} \quad \text { but one : } \bar{D}^{2}\left(\nabla^{2}-D^{2}\right)
\end{array}
$$

For a given graph, i.e. for a fixed number of vertices, the completion of the $D$ algebra is very simple: only one vertex contains $\bar{D}$ 's and the two are both needed in 
$\delta^{4}\left(\theta-\theta^{\prime}\right) \bar{D}^{2} D^{2} \delta^{4}\left(\theta-\theta^{\prime}\right)=\delta^{4}\left(\theta-\theta^{\prime}\right)$. Taking advantage of this covariant technique, the evaluation of the one-loop contributions from chiral superfields has been reduced to a rather trivial exercise.

Now we turn to the noncommutative theory and evaluate explicitly the two- and the three-point function contributions.

The extension of the above procedure to noncommutative super Yang-Mills is straightforward [6, 7]. One simply has to take into proper account the $*$-multiplication which replaces the standard product in the formulas above. This amounts to the introduction of exponential factors in the vertices, while the propagators remain unchanged. In particular at the vertices one has to keep track of the order in which the quantum fields are Wick contracted, a different order giving rise to a different exponential factor and ultimately to a planar or a non planar diagram. In complete generality a vertex quadratic in the quantum fields can be written symbolically in momentum space as

$$
\begin{aligned}
\mathcal{U}\left(k_{1}, k_{2}, k_{3}\right) & =\operatorname{Tr}\left(Q\left(k_{1}\right) * \mathbf{B}\left(k_{2}\right) * Q\left(k_{3}\right)\right) \\
& =\operatorname{Tr}\left(T^{a} T^{b} T^{c}\right) e^{-\frac{i}{2}\left(k_{1} \times k_{2}+k_{2} \times k_{3}+k_{1} \times k_{3}\right)} Q_{a}\left(k_{1}\right) \mathbf{B}_{b}\left(k_{2}\right) Q_{c}\left(k_{3}\right)
\end{aligned}
$$

where $Q$ and $\mathbf{B}$ denote the quantum and the background superfields respectively. The momenta are flowing into the vertex, $k_{1}+k_{2}+k_{3}=0$, and we have used the definition $k_{i} \times k_{j} \equiv\left(k_{i}\right)_{\mu} \Theta^{\mu \nu}\left(k_{j}\right)_{\nu}$ for the $*$-product. Depending on the order in which the quantum $Q$-fields are Wick contracted when inserted in the loop, from each vertex one obtains two types of terms, an untwisted $P$-term and a twisted $T$-term [6, []

$$
\begin{gathered}
P \rightarrow \operatorname{Tr}\left(T^{a} T^{b} T^{c}\right) e^{-\frac{i}{2}\left(k_{1} \times k_{2}+k_{2} \times k_{3}+k_{1} \times k_{3}\right)}=\operatorname{Tr}\left(T^{a} T^{b} T^{c}\right) e^{-\frac{i}{2} k_{2} \times k_{3}} \\
T \rightarrow \pm \operatorname{Tr}\left(T^{c} T^{b} T^{a}\right) e^{\frac{i}{2}\left(k_{1} \times k_{2}+k_{2} \times k_{3}+k_{1} \times k_{3}\right)}= \pm \operatorname{Tr}\left(T^{c} T^{b} T^{a}\right) e^{\frac{i}{2} k_{2} \times k_{3}}
\end{gathered}
$$

The,+- sign in the $T$-term takes into account the bosonic, respectively fermionic, nature of the background external field.

With all of this in mind, we proceed with the following strategy: for each diagram the calculation is performed in momentum space, and first we complete the $D$-algebra in the loop, second we introduce the exponential factors from the $*$-products at the vertices and make a distinction between planar and non planar contributions, finally we evaluate the momentum integral. In order to accomplish the first step we rewrite the vertices in (17) with flat derivatives and explicit dependence on the background (the $*$-multiplication between superfields is always understood)

$$
\begin{aligned}
\square_{+}-\square_{0} & \rightarrow \Delta_{+}-i \mathbf{W}^{\alpha} D_{\alpha} \\
\bar{D}^{2}\left(\nabla^{2}-D^{2}\right) & \rightarrow-i \boldsymbol{\Gamma}^{\alpha} \bar{D}^{2} D_{\alpha}-\frac{1}{2}\left[i\left(D^{\alpha} \boldsymbol{\Gamma}_{\alpha}\right)+\boldsymbol{\Gamma}^{\alpha} \boldsymbol{\Gamma}_{\alpha}\right] \bar{D}^{2}
\end{aligned}
$$


where we have defined

$$
\Delta_{+} \equiv-i \boldsymbol{\Gamma}^{a} \partial_{a}-\frac{i}{2}\left(\partial^{a} \boldsymbol{\Gamma}_{a}\right)-\frac{1}{2} \boldsymbol{\Gamma}^{a} \boldsymbol{\Gamma}_{a}-\frac{i}{2}\left(D^{\alpha} \mathbf{W}_{\alpha}\right)-i \mathbf{W}^{\alpha} \boldsymbol{\Gamma}_{\alpha}
$$

Now we go back to (15) and start expanding the exponentials in order to compute the various contributions to the one-loop effective action:

$$
\begin{array}{ll}
\text { (1) : } & \bar{D}^{2}\left(\nabla^{2}-D^{2}\right)\left(-\frac{1}{\square_{0}}\right) \\
\text { (2) : } \quad\left(\square_{+}-\square_{0}\right)\left(-\frac{1}{\square_{0}}\right) \bar{D}^{2}\left(\nabla^{2}-D^{2}\right)\left(-\frac{1}{\square_{0}}\right) \\
(3): \quad\left(\square_{+}-\square_{0}\right)\left(-\frac{1}{\square_{0}}\right)\left(\square_{+}-\square_{0}\right)\left(-\frac{1}{\square_{0}}\right) \bar{D}^{2}\left(\nabla^{2}-D^{2}\right)\left(-\frac{1}{\square_{0}}\right) \\
\text { (4) : } \quad\left(\square_{+}-\square_{0}\right)\left(-\frac{1}{\square_{0}}\right)\left(\square_{+}-\square_{0}\right)\left(-\frac{1}{\square_{0}}\right)\left(\square_{+}-\square_{0}\right)\left(-\frac{1}{\square_{0}}\right) \bar{D}^{2}\left(\nabla^{2}-D^{2}\right)\left(-\frac{1}{\square_{0}}\right)
\end{array}
$$

Since at least two $D$ 's and two $\bar{D}$ 's are needed for a nonzero result, from (20) we immediately obtain that the first order expansion does not contribute. Moreover since the vertex $\left(\square_{+}-\square_{0}\right)$ contains at most one $D$ that can be used for the $D$-algebra, the second order expansion only contributes to the two-point function

$$
\begin{aligned}
\left(\square_{+}-\square_{0}\right)\left(-\frac{1}{\square_{0}}\right) \bar{D}^{2}\left(\nabla^{2}-D^{2}\right)\left(-\frac{1}{\square_{0}}\right) & \rightarrow-i \mathbf{W}^{\alpha} D_{\alpha}\left(-\frac{1}{\square_{0}}\right)\left(-i \boldsymbol{\Gamma}^{\beta} \bar{D}^{2} D_{\beta}\right)\left(-\frac{1}{\square_{0}}\right) \\
& \rightarrow \mathbf{W}^{\alpha}\left(-\frac{1}{\square_{0}}\right) \boldsymbol{\Gamma}_{\alpha}\left(-\frac{1}{\square_{0}}\right)
\end{aligned}
$$

According to the general strategy, having completed the $D$-algebra we have to take into account the nature of the quantum background vertices we started with. Going back to (19), we learn that the combination $P P+T T$ corresponds to the planar graphs, while $P T+T P$ gives rise to the non planar ones. Thus we obtain the following contributions to the one-loop two-point function:

$$
\begin{aligned}
\Gamma_{\text {planar }}^{(2)}=N \int d^{2} \theta \frac{d^{4} p}{(2 \pi)^{4}} & \operatorname{Tr}\left(\mathbf{W}^{\alpha}(p) \mathbf{W}_{\alpha}(-p)\right) \\
& \int \frac{d^{4} k}{(2 \pi)^{4}} \frac{1}{\left(k^{2}+m^{2}\right)\left[(p+k)^{2}+m^{2}\right]}
\end{aligned}
$$

and

$$
\begin{array}{r}
\Gamma_{\text {nonplanar }}^{(2)}=-\int d^{2} \theta \frac{d^{4} p}{(2 \pi)^{4}} \operatorname{Tr}\left(\mathbf{W}^{\alpha}(p)\right) \operatorname{Tr}\left(\mathbf{W}_{\alpha}(-p)\right) \\
\int \frac{d^{4} k}{(2 \pi)^{4}} \frac{e^{i p \times k}}{\left(k^{2}+m^{2}\right)\left[(p+k)^{2}+m^{2}\right]}
\end{array}
$$


where $m$ is an IR mass regulator. The result in (24) reproduces the logarithmic UV divergence that leads to a nonvanishing one-loop $\beta$-function both for the $\mathcal{N}=1$ and the $\mathcal{N}=2$ theories, with the counting that we already mentioned, i.e. $(-3)$ times the contribution in (24) from the three ghosts in the $\mathcal{N}=1$ case, $(-3+1)$ from the three ghosts and one chiral matter for the $\mathcal{N}=2$ case.

The corresponding nonplanar result in (25) is finite in the UV thanks to the presence of the non local scale $\Delta x^{\mu}=\Theta^{\mu \nu} p_{\nu}$ that acts as a short distance cutoff. In the low-energy approximation, $p_{i} \cdot p_{j}$ small, with $p_{i} \times p_{j}$ finite it is easy to perform the $k$-integration. With the definition $p \circ p=p_{\mu} \Theta^{\mu \nu} \Theta_{\rho \nu} p^{\rho}$, one obtains

$$
\Gamma_{\text {nonplanar }}^{(2) l . e .}=-\frac{1}{8 \pi^{2}} \int d^{2} \theta \frac{d^{4} p}{(2 \pi)^{4}} \operatorname{Tr}\left(\mathbf{W}^{\alpha}(p)\right) \operatorname{Tr}\left(\mathbf{W}_{\alpha}(-p)\right) K_{0}(m \sqrt{p \circ p})
$$

Since

$$
\lim _{z \rightarrow 0} K_{0}(z)=-\ln \frac{z}{2}
$$

the expression in (26) is divergent in the IR [5]. In a sense the real novelty of noncommutative theories is given by the interplay between the UV and the IR behaviour. The lack of conformal invariance of the quantum commutative theory $(\beta \neq 0)$ leads to a nonperturbative, singular behaviour in the non local scale $(\Theta \neq 0)$. A complete understanding of the role played by this effect is likely to be found from string dynamics 15.

Now we continue in our task and turn to the computation of the vector three-point function due to a chiral matter loop.

As observed before, the second order expansion in (22) contributes only to the two-point function. Thus the three-point function is obtained from the third order terms

$$
\left(\square_{+}-\square_{0}\right)\left(-\frac{1}{\square_{0}}\right)\left(\square_{+}-\square_{0}\right)\left(-\frac{1}{\square_{0}}\right) \bar{D}^{2}\left(\nabla^{2}-D^{2}\right)\left(-\frac{1}{\square_{0}}\right)
$$

where at every vertex we only keep terms which are linear in the background fields. Inserting (20) and (21) in (28) we obtain several contributions, all with trivial $D$-algebra, that we list:

$$
\begin{gathered}
\text { (a) : } \quad\left(-i \mathbf{W}^{\gamma} D_{\gamma}\right)\left(-\frac{1}{\square_{0}}\right)\left(-i \mathbf{W}^{\beta} D_{\beta}\right)\left(-\frac{1}{\square_{0}}\right) \bar{D}^{2}\left(-\frac{i}{2} D^{\alpha} \boldsymbol{\Gamma}_{\alpha}\right)\left(-\frac{1}{\square_{0}}\right) \\
\longrightarrow \quad \mathbf{W}^{\beta}\left(-\frac{1}{\square_{0}}\right) \mathbf{W}_{\beta}\left(-\frac{1}{\square_{0}}\right)\left(-\frac{i}{2} D^{\alpha} \boldsymbol{\Gamma}_{\alpha}\right)\left(-\frac{1}{\square_{0}}\right) \\
(\mathrm{b}): \quad\left(-i \mathbf{W}^{\gamma} D_{\gamma}\right)\left(-\frac{1}{\square_{0}}\right)\left(-i \mathbf{W}^{\beta} D_{\beta}\right)\left(-\frac{1}{\square_{0}}\right)\left(-i \boldsymbol{\Gamma}^{\alpha} \bar{D}^{2} D_{\alpha}\right)\left(-\frac{1}{\square_{0}}\right) \\
\longrightarrow \quad\left(-\mathbf{W}^{\gamma}\right)\left(-\frac{1}{\square_{0}}\right)\left(D_{\gamma} \mathbf{W}^{\beta}\right)\left(-\frac{1}{\square_{0}}\right)\left(i \boldsymbol{\Gamma}_{\beta}\right)\left(-\frac{1}{\square_{0}}\right) \\
+\mathbf{W}^{\beta}\left(-\frac{1}{\square_{0}}\right) \mathbf{W}_{\beta}\left(-\frac{1}{\square_{0}}\right)\left(i D^{\alpha} \boldsymbol{\Gamma}_{\alpha}\right)\left(-\frac{1}{\square_{0}}\right)
\end{gathered}
$$




$$
\begin{gathered}
\text { (c) : } \quad\left[-i \boldsymbol{\Gamma}^{a} \partial_{a}-\frac{i}{2}\left(\partial^{a} \boldsymbol{\Gamma}_{a}\right)-\frac{i}{2} D^{\alpha} \mathbf{W}_{\alpha}\right]\left(-\frac{1}{\square_{0}}\right)\left(-i \mathbf{W}^{\beta} D_{\beta}\right)\left(-\frac{1}{\square_{0}}\right)\left(-i \boldsymbol{\Gamma}^{\gamma} \bar{D}^{2} D_{\gamma}\right)\left(-\frac{1}{\square_{0}}\right) \\
\longrightarrow \quad\left[-i \boldsymbol{\Gamma}^{a} \partial_{a}-\frac{i}{2}\left(\partial^{a} \boldsymbol{\Gamma}_{a}\right)-\frac{i}{2} D^{\alpha} \mathbf{W}_{\alpha}\right]\left(-\frac{1}{\square_{0}}\right) \mathbf{W}^{\beta}\left(-\frac{1}{\square_{0}}\right) \boldsymbol{\Gamma}_{\beta}\left(-\frac{1}{\square_{0}}\right) \\
(\mathrm{d}): \quad\left(-i \mathbf{W}^{\beta} D_{\beta}\right)\left(-\frac{1}{\square_{0}}\right)\left[-i \boldsymbol{\Gamma}^{a} \partial_{a}-\frac{i}{2}\left(\partial^{a} \boldsymbol{\Gamma}_{a}\right)-\frac{i}{2} D^{\alpha} \mathbf{W}_{\alpha}\right]\left(-\frac{1}{\square_{0}}\right)\left(-i \boldsymbol{\Gamma}^{\gamma} \bar{D}^{2} D_{\gamma}\right)\left(-\frac{1}{\square_{0}}\right) \\
\longrightarrow \quad \mathbf{W}^{\beta}\left(-\frac{1}{\square_{0}}\right)\left[-i \boldsymbol{\Gamma}^{a} \partial_{a}-\frac{i}{2}\left(\partial^{a} \boldsymbol{\Gamma}_{a}\right)-\frac{i}{2} D^{\alpha} \mathbf{W}_{\alpha}\right]\left(-\frac{1}{\square_{0}}\right) \boldsymbol{\Gamma}_{\beta}\left(-\frac{1}{\square_{0}}\right)
\end{gathered}
$$

Having completed the $D$-algebra now we have to take into account the $*$-products and the orderings from Wick contractions at the vertices. We have external momenta $p_{1}, p_{2}$, $p_{3}$ with $p_{1}+p_{2}+p_{3}=0$ and a momentum $k$ in the loop. Planar diagrams correspond to arrangements of the vertices like $P_{1} P_{2} P_{3}$ and $T_{1} T_{2} T_{3}$, while non planar diagrams correspond to the six terms $P_{1} P_{2} T_{3}, P_{1} T_{2} P_{3}, T_{1} P_{2} P_{3}, T_{1} T_{2} P_{3}, T_{1} P_{2} T_{3}, P_{1} T_{2} T_{3}$. In order to write the final result in a simple form it is convenient to arrange the external background fields in the most symmetric way, i.e. for every term we write

$$
\mathbf{A}_{a}\left(p_{1}\right) \mathbf{B}_{b}\left(p_{2}\right) \mathbf{C}_{c}\left(p_{3}\right)+\text { all permutations }\left(\mathrm{p}_{1} \mathrm{a}\right) \leftrightarrow\left(\mathrm{p}_{2} \mathrm{~b}\right) \leftrightarrow\left(\mathrm{p}_{3} \mathrm{c}\right)
$$

Now we are ready to analyze the contributions in (29). We can use the symmetry property in (30) and exchange the external fields (keeping track of the correct sign when fermionic fields are involved). Also we can integrate by parts derivatives in order to reduce the various terms to similar structures. In this way one finds immediately that the contributions containing the connection $\boldsymbol{\Gamma}_{a}$ cancel. In fact making use of the relation

$$
D_{\alpha} \mathbf{W}_{\beta}=\frac{1}{2} D_{(\alpha} \mathbf{W}_{\beta)}+\frac{1}{2} C_{\beta \alpha} D^{\gamma} \mathbf{W}_{\gamma}
$$

it is easy to show that also the remaining terms add up to zero. Thus the chiral matter contribution to the vector three-point function is zero. This result, combined with the fact that vectors circulating in the loop do not contribute (not enough $D$ 's in the loop), leads to the conclusion that the one-loop three-point function with vector external lines is zero for $\mathcal{N}=1, \mathcal{N}=2$ and $\mathcal{N}=4$ supersymmetric Yang-Mills. The simplicity of the above calculation is a good example of the power of the background covariant superfield approach as compared to standard superfield techniques (cf. [16]) or to component field methods.

Finally we consider the chiral loop corrections to the effective action with four vector external fields. Here we explain the general procedure while the details and the presentation of the complete answer will be given elsewhere [17].

One obtains contributions from the third order expansion in (22) with one of the vertices quadratic in the background, and contributions from the fourth order expansion 
with all the vertices linear in the background. It is straightforward to list the various terms and perform the $D$-algebra, exactly as we have done in the previous two examples. Once again one can exploit the symmetries of the four-point function, i.e. for every term we write

$$
\mathbf{A}_{a}\left(p_{1}\right) \mathbf{B}_{b}\left(p_{2}\right) \mathbf{C}_{c}\left(p_{3}\right) \mathbf{D}_{d}\left(p_{4}\right)+\text { all permutations }\left(\mathrm{p}_{1} \mathrm{a}\right) \leftrightarrow\left(\mathrm{p}_{2} \mathrm{~b}\right) \leftrightarrow\left(\mathrm{p}_{3} \mathrm{c}\right) \leftrightarrow\left(\mathrm{p}_{4} \mathrm{~d}\right)
$$

In this way the result is drastically simplified and one can isolate the terms which, at the component level, give rise to structures with four external $F_{\mu \nu}$ field strengths. To this end one has to remember that the bosonic field strength $F_{\mu \nu}$ is contained in the superfield [1]

$$
D_{(\alpha} \mathbf{W}_{\beta)} \rightarrow f_{\alpha \beta}=\sigma_{\alpha \beta}^{\mu \nu} F_{\mu \nu}
$$

where $\left(\sigma_{\mu \nu}\right)_{\alpha}^{\beta} \equiv-1 / 4\left(\sigma_{\mu} \tilde{\sigma}_{\nu}-\sigma_{\nu} \tilde{\sigma}_{\mu}\right)_{\alpha}^{\beta}$ and $\left(\sigma_{\mu}\right)_{\alpha \dot{\alpha}} \equiv(1, \vec{\sigma}),\left(\tilde{\sigma}_{\nu}\right)^{\dot{\alpha} \alpha} \equiv(1,-\vec{\sigma})$. Back to the four-point function, one finds relevant contributions in the form

$$
\mathbf{W}_{\gamma}\left(-\frac{1}{\square_{0}}\right)\left(D^{\beta} \mathbf{W}^{\gamma}\right)\left(-\frac{1}{\square_{0}}\right)\left(D_{\beta} \mathbf{W}^{\alpha}\right)\left(-\frac{1}{\square_{0}}\right) \boldsymbol{\Gamma}_{\alpha}\left(-\frac{1}{\square_{0}}\right)
$$

The external fields, completely symmetrized, are arranged as follows

$$
\begin{aligned}
\mathcal{R}(1 a, 2 b, 3 c, 4 d) & \equiv \mathbf{W}_{a \gamma}\left(p_{1}\right)\left(D^{\beta} \mathbf{W}_{b}^{\gamma}\right)\left(p_{2}\right)\left(D_{\beta} \mathbf{W}_{c}^{\alpha}\right)\left(p_{3}\right) \boldsymbol{\Gamma}_{d \alpha}\left(p_{4}\right)+ \\
& + \text { all permutations }\left(\mathrm{p}_{1} \mathrm{a}\right) \leftrightarrow\left(\mathrm{p}_{2} \mathrm{~b}\right) \leftrightarrow\left(\mathrm{p}_{3} \mathrm{c}\right) \leftrightarrow\left(\mathrm{p}_{4} \mathrm{~d}\right)
\end{aligned}
$$

so that the above expression can be compared directly with the bosonic component amplitude since

$$
\int d^{4} \theta \mathcal{R}(1 a, 2 b, 3 c, 4 d) \rightarrow t^{\mu_{1} \nu_{1} \mu_{2} \nu_{2} \mu_{3} \nu_{3} \mu_{4} \nu_{4}} F_{\mu_{1} \nu_{1}}^{a}\left(p_{1}\right) F_{\mu_{2} \nu_{2}}^{b}\left(p_{2}\right) F_{\mu_{3} \nu_{3}}^{c}\left(p_{3}\right) F_{\mu_{4} \nu_{4}}^{d}\left(p_{4}\right)
$$

where $t^{\mu_{1} \nu_{1} \mu_{2} \nu_{2} \mu_{3} \nu_{3} \mu_{4} \nu_{4}}$ is the symmetric tensor given e.g. in (9.A.18) of [18].

In addition to terms like the ones in (34), one obtains also terms in the form

$$
\mathbf{W}^{\alpha}\left(-\frac{1}{\square_{0}}\right) \mathbf{W}_{\alpha}\left(-\frac{1}{\square_{0}}\right) \overline{\mathbf{W}}^{\dot{\alpha}}\left(-\frac{1}{\square_{0}}\right) \overline{\mathbf{W}}_{\dot{\alpha}}\left(-\frac{1}{\square_{0}}\right)
$$

which are generated by the appearance of structures like $i \partial_{a} \boldsymbol{\Gamma}^{\alpha}-i D^{\alpha} \boldsymbol{\Gamma}_{a}=2 \overline{\mathbf{W}}_{\dot{\alpha}}$. Using the relation

$$
\begin{aligned}
& \int d^{4} \theta\left[\mathbf{W}^{\alpha a}\left(p_{1}\right) \mathbf{W}_{\alpha}^{b}\left(p_{2}\right) \overline{\mathbf{W}}^{\dot{\alpha} c}\left(p_{3}\right) \overline{\mathbf{W}}_{\dot{\alpha}}^{d}\left(p_{4}\right)\right. \\
& \left.+ \text { all permutations }\left(\mathrm{p}_{1} \mathrm{a}\right) \leftrightarrow\left(\mathrm{p}_{2} \mathrm{~b}\right) \leftrightarrow\left(\mathrm{p}_{3} \mathrm{c}\right) \leftrightarrow\left(\mathrm{p}_{4} \mathrm{~d}\right)\right] \\
& \rightarrow t^{\mu_{1} \nu_{1} \mu_{2} \nu_{2} \mu_{3} \nu_{3} \mu_{4} \nu_{4}} F_{\mu_{1} \nu_{1}}^{a}\left(p_{1}\right) F_{\mu_{2} \nu_{2}}^{b}\left(p_{2}\right) F_{\mu_{3} \nu_{3}}^{c}\left(p_{3}\right) F_{\mu_{4} \nu_{4}}^{d}\left(p_{4}\right)
\end{aligned}
$$


from the superfield result in (37) once again one can extract the bosonic contributions.

Then in all the various terms one has to insert the appropriate $*$-products that give rise to planar and non planar diagrams, exactly as it has been done in [6, 7]. At that stage one evaluates the loop momentum integrals with four propagators

$$
I_{0}\left(k ; p_{1}, \ldots, p_{4}\right)=\frac{1}{\left[\left(k+p_{1}\right)^{2}+m^{2}\right]\left[k^{2}+m^{2}\right]\left[\left(k-p_{4}\right)^{2}+m^{2}\right]\left[\left(k+p_{1}+p_{2}\right)^{2}+m^{2}\right]}
$$

for each diagram.

Once all the terms have been collected, again as we have argued for the two-point function, we have to multiply the final answer by $(-3)$ from the three chiral ghosts in the case of the $\mathcal{N}=1$ theory, while for $\mathcal{N}=2$ we have the same contribution times the factor $(-3+1)$ which counts the three ghosts and one chiral superfield. The complete four-point function with vector external fields [17] is then obtained by adding to the oneloop matter corrections the corresponding one-loop vector corrections computed in [7]. It would be interesting to evaluate the corresponding amplitudes in the appropriate D3brane configurations and compare the field theory limit from the string approach to the one-loop field theory effective action calculations.

A neat result that we have obtained is the vanishing of the vector three-point function in all $\mathcal{N}=1,2,4$ supersymmetric Yang-Mills theories. Since we have used the background field quantization this result is a gauge invariant statement. It is in correspondence with the fact that in the supersymmetric Born-Infeld action terms cubic in the $F_{\mu \nu}$ field strength are absent.

The superfield background covariant method provides a very efficient mean of computing in perturbation theory. One could take advantage of this and study renormalization properties of correlators of gauge invariant operators in noncommutative field theories [19, 20].

\section{Acknowledgements}

I thank Marc Grisaru and Carlos Nunez for interesting conversations and the Department of Harvard University for warm hospitality while this work has been performed. This work has been partially supported by INFN, MURST, and the European Commission RTN program HPRN-CT-2000-00113 in which I am associated to the University of Torino. 


\section{References}

[1] A. Connes, M.R. Douglas, A. Schwarz, JHEP 02 (1998) 003, hep-th/9711162

M.R. Douglas, C. Hull, JHEP 02 (1998) 008, hep-th/9711165

[2] N. Seiberg, E. Witten, JHEP 09 (1999) 032, hep-th/9908142

[3] N. Seiberg, L. Susskind, N. Toumbas, JHEP 06 (2000) 021, hep-th/000540

R. Gopakumar, J. Maldacena, S. Minwalla, A. Strominger, JHEP 06 (2000) 036, hep-th/0005048

R. Gopakumar,S. Minwalla, N. Seiberg, A. Strominger, JHEP 08 (2000) 008, hepth/0006062

E. Bergshoeff, D.S. Berman, J.P. van der Schaar, P. Sundell, Phys. Lett. B492 (2000) 193, hep-th/0006112

[4] O. Andreev, H. Dorn, Nucl. Phys. B583 (2000) 145, hep-th/0003113

A. Bilal, C.-S. Chu, R. Russo, Nucl. Phys. B582 (2000) 65, hep-th/0003180

C.-S. Chu, R. Russo, S. Sciuto, Nucl.Phys. B585 (2000) 193, hep-th/0004183

[5] T. Filk, Phys. Lett. B376(1996) 53

S. Minwalla, M. Van Raamsdonk, N. Seiberg, hep-th/9912072

A. Matusis, L. Susskind, N. Toumbas, hep-th/0002075

M. Van Raamsdonk, N. Seiberg, JHEP 03 (2000) 035, hep-th/0002186

M.M. Sheikh-Jabbari, JHEP 9906 (1999) 015, hep-th/9903107

C.P. Martin, D. Sanchez-Ruiz, Phys. Rev. Lett. 83 (1999) 476, hep-th/9903077

T. Krajewski, R. Wulkenhaar, J. Mod. Phys. A15 (2000) 1011, hep-th/9903187

I.Y. Arefeva, D.M. Belov, A.S. Koshelev, O.A. Rychkov, Phys. Lett. B487 (2000) 357, hep-th/0003176

C.P. Martin, F. Ruiz Ruiz, hep-th/0007131

I. Chepelev, R. Roiban, JHEP 05 (2000) 037, hep-th/9911098; hep-th/0008090

M. Haykawa, Phys. Lett. B478 (2000) 394, hep-th/9912094; hep-th/9912167

A. Armoni, hep-th/0005208

V.V. Khoze, G. Travaglini, hep-th/0011218

[6] D. Zanon, hep-th/0009196

[7] A. Santambrogio, D. Zanon, hep-th/0010275

M. Pernici, A. Santambrogio, D. Zanon, hep-th/0011140

[8] S. Ferrara, M.A. Lledo', JHEP 05 (2000) 008, hep-th/0002084

[9] M.T. Grisaru, W. Siegel, Nucl. Phys. B201 (1982) 292

[10] M.T. Grisaru, W. Siegel, Phys. Lett. 110B (1982) 49

[11] S.J. Gates, M.T. Grisaru, M. Rocek, W. Siegel, "Superspace" (Benjamin-Cummings, Reading, MA, 1983) 
[12] H. Liu, J. Michelson, hep-th/0008205

[13] M.T. Grisaru, D. Zanon, Nucl. Phys B252 (1985) 578

[14] L. Bonora, M. Schnabl, M.M. Sheikh-Jabbari, A. Tomasiello, Phys. Lett. B485 (2000) 311, hep-th/0002210; Nucl. Phys. B589 (2000) 461, hep-th/0006091

C.P. Martin, hep-th/0008126

F. Ardalan, N. Sadooghi, hep-th/0009233

M.T. Grisaru, S. Penati, hep-th/0010177

[15] H. Liu, J. Michelson, Phys. Rev. D62 (2000) 066003, hep-th/0004013

[16] W.E. Caswell, D. Zanon, Nucl. Phys. B182 (1981) 125

[17] D. Zanon, in preparation

[18] M.B. Green, J.H. Schwarz, E. Witten, "Superstring Theory" (Cambridge University Press, 1987)

[19] S. Das, S.-J. Rey, hep-th/0008042

D.J. Gross, A. Hashimoto, N. Itzhaki, hep-th/0008075

T. Mehen, M.B. Wise, hep-th/0010204

H. Liu, hep-th/0011125

[20] N. Ishibashi, S. Iso, H. Kawai, Y. Kitazwa, Nucl. Phys. B573 (2000) 573, hepth/9910004 